\title{
Poor Man's Scaling and Green Function Analysis of the Kondo Anomaly in Single-Level Quantum Dots
}

\author{
M. WAWRZYNIAK ${ }^{a}$, R. Ś'WIRKOWICZ ${ }^{b}$, M. WILCZYŃSKI ${ }^{b}$ \\ AND J. BARNAŚ ${ }^{a, c}$ \\ ${ }^{a}$ Department of Physics, Adam Mickiewicz University \\ Umultowska 85, 61-614 Poznań, Poland \\ ${ }^{b}$ Faculty of Physics, Warsaw University of Technology \\ Koszykowa 75, 00-662 Warsaw, Poland \\ ${ }^{c}$ Institute of Molecular Physics, Polish Academy of Sciences \\ Smoluchowskiego 17, 60-179 Poznań, Poland
}

Kondo effect in a single-level quantum dot attached to magnetic leads is studied theoretically by the "poor man's scaling" and non-equilibrium Green function methods. From the scaling equations we derive the Kondo temperature as a function of the model parameters - in particular as a function of the angle between magnetic moments. Transport characteristics, i.e. differential conductance and tunnel magnetoresistance associated with magnetization rotation, were calculated within the non-equilibrium Green function formalism based on the equation of motion method.

PACS numbers: 75.20.Hr, 72.15.Qm, 72.25.-b, 73.23.Hk

\section{Introduction}

Kondo effect in quantum dots attached to metallic leads (electrodes) is currently a subject of extensive investigations. In most of the relevant works, the authors dealt with non-magnetic leads, where the Kondo anomaly in differential conductance is clearly seen below a certain (Kondo) temperature [1-3]. The Kondo effect was studied by several different theoretical techniques, including the "poor man's scaling", renormalization group, nanoequilibrium Green function technique, and others. Significantly less attention was paid to the case of ferromagnetic leads. It was shown recently for collinear configurations of the leads' magnetic moments that the electrodes' ferromagnetism may lead to interesting new phenomena in the Kondo problem, which are absent in the case of non-magnetic leads [4-6]. The most important one is the suppression of the Kondo anomaly in the parallel magnetic configuration due to an exchange field generated by the spin dependent dot-electrodes coupling. 
In this work, we consider the Kondo phenomenon in the case of non-collinear magnetic moments. Such a configurations was studied in Ref. [7] by the Green functions technique in the infinite $U$ limit, and more recently also by the "poor man's scaling" and renormalization group techniques $[8,9]$.

\section{Model}

We consider a single-level quantum dot symmetrically coupled to ferromagnetic electrodes. The magnetic moments of the electrodes are in general noncollinear [7]. The system is described by the Anderson-like Hamiltonian of the general form $H_{\mathrm{A}}=H_{\mathrm{L}}+H_{\mathrm{R}}+H_{\mathrm{D}}+H_{\mathrm{T}}$. The terms $H_{\alpha}(\alpha=\mathrm{L}, \mathrm{R})$ describe non-interacting electrons in the left and right electrodes, respectively. The term $H_{\mathrm{D}}=\sum_{\sigma} \epsilon_{\sigma}^{\mathrm{d}} d_{\sigma}^{\dagger} d_{\sigma}+U d_{\uparrow}^{\dagger} d_{\uparrow} d_{\downarrow}^{\dagger} d_{\downarrow}$ describes the dot, where $\epsilon_{\sigma}^{\mathrm{d}}$ is the single-particle energy of the dot's level, whereas $U$ is the Coulomb correlation parameter. The dot's level $\epsilon_{\sigma}^{\mathrm{d}}$ includes the Zeeman energy due to external magnetic field $B_{\text {ext }}^{\mathrm{z}}$ applied along the dot's quantization axis. The tunnelling term $H_{\mathrm{T}}$ describes electron hopping between the dot and electrodes and has the form

$$
H_{\mathrm{T}}=\sum_{\boldsymbol{k}_{\alpha}} \sum_{\beta \sigma} T_{\alpha \boldsymbol{k} \beta} a_{\alpha \boldsymbol{k} \beta}^{\dagger} R_{\alpha \beta \sigma} d_{\sigma}+\text { h.c., }
$$

with $T_{\alpha \boldsymbol{k} \beta}$ denoting the tunneling matrix elements, and $\boldsymbol{R}_{\alpha}$ being the relevant spin rotation matrix (corresponding to the angle $\phi_{\alpha}$ between the quantization axis on the dot and the local quantization axis in the $\alpha$-th electrode). Electron spin projection on the dot's quantization axis is denoted by $\sigma=\uparrow(\downarrow)$, while the spin projection on the local quantization axis in the electrodes is denoted by $\beta=+(-)$. The coupling strength is described by $\Gamma_{\alpha \beta}(\epsilon)=2 \pi \sum_{\boldsymbol{k}}\left|T_{\alpha \boldsymbol{k} \beta}\right|^{2} \delta\left(\epsilon-\epsilon_{\alpha \boldsymbol{k} \beta}\right)$ and is assumed to be energy independent within the energy band extending from $-D$ to $D, \Gamma_{\alpha \beta}(\epsilon)=\Gamma_{\alpha \beta}=\Gamma_{\alpha}^{0}\left(1 \pm p_{\alpha}\right)$, where $p_{\alpha}$ is the spin polarization of the $\alpha$-th electrode. We also define $\Delta=\sum_{\alpha} \Gamma_{\alpha}^{0}$ and assume $\Gamma_{\mathrm{L}}^{0}=\Gamma_{\mathrm{R}}^{0}=\Gamma^{0}, p_{\mathrm{L}}=p_{\mathrm{R}}=p$. In the following we restrict our consideration to the symmetrical situation, i.e. for $-\phi_{\mathrm{L}}=\phi_{\mathrm{R}}=\theta / 2$.

\section{3. "Poor man's scaling" approach}

In order to analyze the Kondo temperature and its dependence on the model parameters, we applied the "poor man's scaling" approach to the model Hamiltonian describing the quantum dot coupled to non-collinearly polarized magnetic leads. We assume that the dot is initially singly occupied, i.e. $-D \ll \epsilon_{\sigma}^{\mathrm{d}} \ll \mu$ and $\epsilon_{\sigma}^{\mathrm{d}}+U \gg \mu_{\alpha}$, where $\mu(\mu=0)$ is the chemical level of the electrodes. Following the general rules of the scaling approach [1], we reduce first the cut-off (band width) by integrating out high-energy fluctuations in the conduction electron band. This procedure takes into account spin-dependent charge fluctuations which lead to renormalization of the Hamiltonian parameters, and in particular to spin splitting $\delta \epsilon=\epsilon_{\uparrow}^{\mathrm{d}}-\epsilon_{\downarrow}^{\mathrm{d}}$ of the bare dot level $\epsilon^{\mathrm{d}}$. The scaling of the Anderson Hamiltonian is terminated when the cut-off $\tilde{D}$ approaches the dot level, where the 

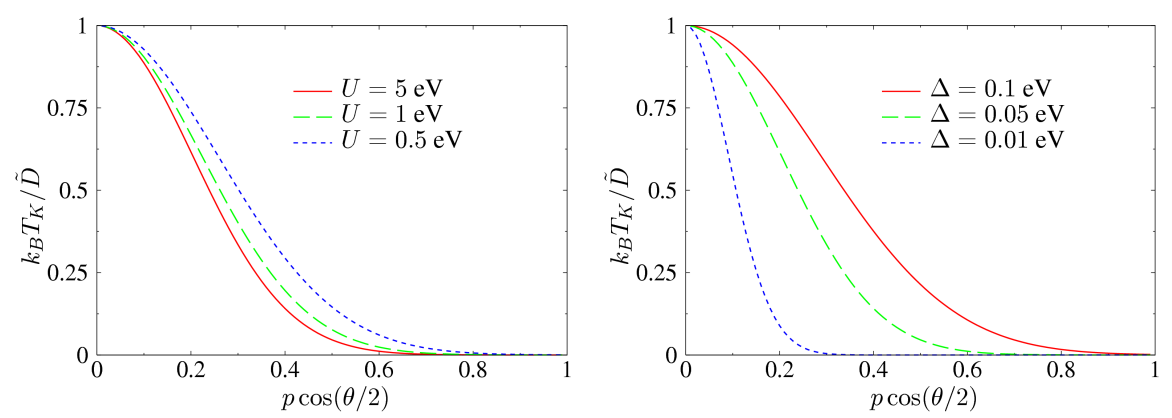

Fig. 1. The Kondo temperature vs. $p \cos (\theta / 2)$, for $p=0.5, \epsilon^{\mathrm{d}}=-0.2 \mathrm{eV}, D=25 \mathrm{eV}$, $U=5 \mathrm{eV}$ (right), and $\Delta=0.1 \mathrm{eV}$ (left). The other parameters as indicated.
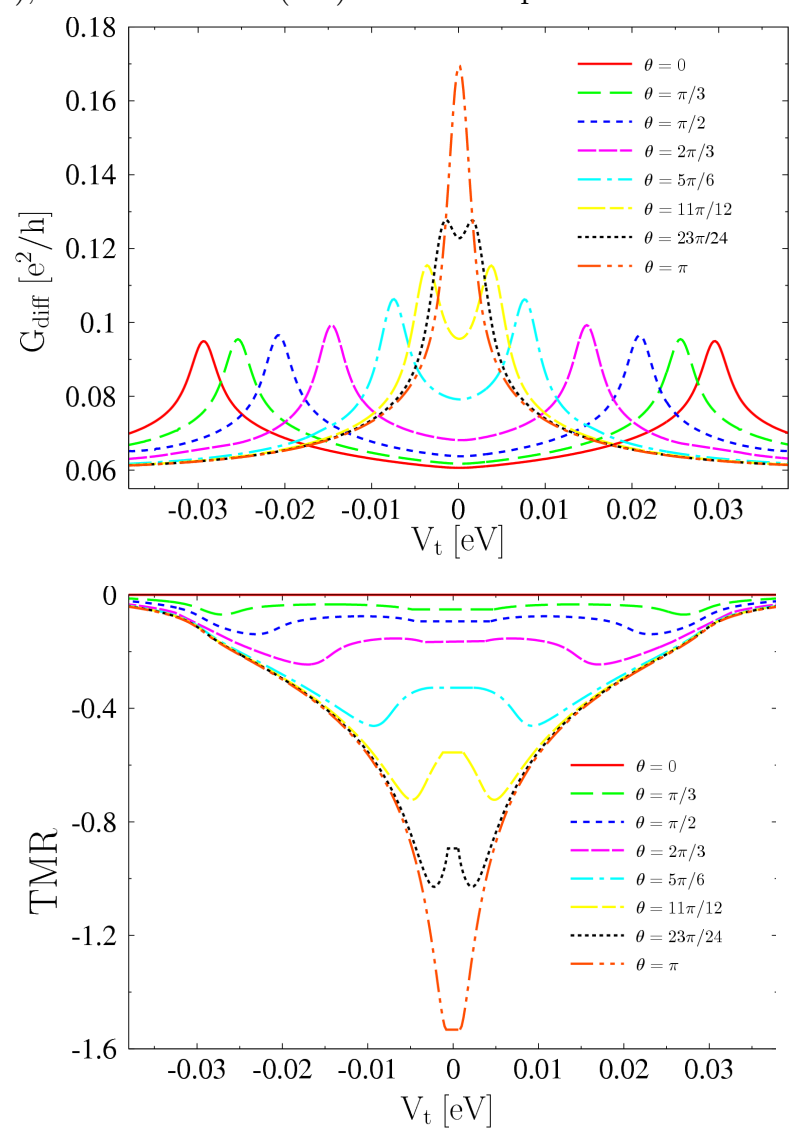

Fig. 2. Differential conductance (top) and tunneling magnetoresistance (bottom) vs. bias voltage $V_{\mathrm{t}}$ for a few magnetic configurations, and for $\epsilon^{\mathrm{d}}=-0.45 \mathrm{eV}, U=5 \mathrm{eV}$, $\Gamma^{0}=0.1 \mathrm{eV}, k_{\mathrm{B}} T=0.001 \mathrm{eV}, p=0.2$.

perturbation theory breaks down. Then the Schrieffer-Wolff transformation [10] is applied leading to an effective $s-d$ (Kondo) Hamiltonian. The scaling procedure 
is then continued, but now for the Kondo Hamiltonian. Writing down the scaling equations and analyzing the corresponding fixed point, one arrives at the following expression for the Kondo temperature $T_{\mathrm{K}}$ :

$$
T_{\mathrm{K}}(p, \theta)=\tilde{D} \exp \left(-\frac{\operatorname{arctanh}(p \cos (\theta / 2))}{2 j_{0} p \cos (\theta / 2)}\right),
$$

with $j_{0}=(\Delta / \pi)\left(U /\left(\left|\epsilon^{\mathrm{d}}\right|\left(U+\epsilon^{\mathrm{d}}\right)\right)\right.$. This equation was derived first in Refs. [8, 9]. The corresponding numerical data are shown in Fig. 1, where the Kondo temperature is plotted as a function of the parameter $p \cos (\theta / 2)$. This figure clearly shows that the Kondo temperature goes to zero when $p \rightarrow 1$ and magnetic configuration tends to parallel alignment.

\section{Equation of motion method}

To find conductance of the system we applied the equation of motion method for the non-equilibrium Green function. The electric current has been calculated following Ref. [2], but additionally the effective exchange magnetic field $B_{\text {ex }}$ arising in non-collinear systems due to coupling with ferromagnetic electrodes [7] has been taken into account. This exchange field is responsible for the reduction of the Kondo anomaly in differential conductance, as shown in Fig. 2, where the conductance as well as the corresponding tunnel magnetoresistance are shown for several values of the angle between magnetic moments of the leads. Suppression of the Kondo anomaly is clearly seen when the configuration departs from the antiparallel alignment. This suppression leads to negative tunnel magnetoresistance.

\section{Acknowledgments}

This work is supported by funds from the Polish Ministry of Science and Higher Education as a research project in years 2006-2008.

\section{References}

[1] A.C. Hewson, The Kondo Problem to Heavy Fermions, Cambridge University Press, 1993, p. 65.

[2] Y. Meir, N.S. Wingreen, P.A. Lee, Phys. Rev. lett. 70, 2601 (1993).

[3] A. Kaminski, Yu.V. Nazarov, L.I. Glanzman, Phys. Rev. B 62, 8154 (2000).

[4] Y. Utsumi, H. Imamura, J. Barnaś, S. Maekawa, G. Schoen, Phys. Rev. Lett. 91, 127203 (2003).

[5] R. Lopez, D. Sanchez, Phys. Rev. Lett. 90, 116602 (2003).

[6] R. Świrkowicz, M. Wilczyński, M. Waewrzyniak, J. Barnaś, Phys. Rev. B 73, 193312 (2006).

[7] R. Świrkowicz, M. Wilczyński, J. Barnaś, J. Phys., Condens. Matter 18, 2291 (2006).

[8] P. Simon, P.S. Cornaglia, D. Feinberg, C.S. Baleairo, Phys. Rev. B 75, 045310 (2007).

[9] D. Matsubayashi, M. Eto, arXiv.org:cond-mat/0607548.

[10] J.R. Schrieffer, P.A. Wolff, Phys. Rev. 149, 491 (1966). 